\title{
Asistencia oficial para el desarrollo, capital social y crecimiento en América Latina
}

\author{
Isabel Neira, Maricruz Lacalle-Calderón y Marta Portela
}

RESUMEN

En este trabajo se examina la relación entre la asistencia oficial para el desarrollo (AOD),

el capital social y el crecimiento económico en América Latina, con miras a determinar

si el efecto de dicha asistencia en el crecimiento depende de las reservas de capital social del país receptor. Con ese objetivo se utiliza la "confianza" para medir el capital social en un panel incompleto de 18 países latinoamericanos en el período 2001-2010. Después de explicar los efectos relativos al país y al tiempo en un modelo de datos de panel dinámico, los resultados permiten ver que el efecto de la AOD en el crecimiento depende, de hecho, del nivel de confianza que existe. Esto sugiere que esa asistencia será más eficaz cuando se utilice en un ambiente donde dicho nivel sea elevado.

PALABRAS CLAVE

CLASIFICACIÓN JEL

AUTORES
Desarrollo económico, asistencia al desarrollo, crecimiento económico, capital social, modelos econométricos, América Latina

C230, F350, O200, O400

Isabel Neira es Profesora Titular de Econometría en la Facultad de Ciencias Económicas y Empresariales de la Universidad de Santiago de Compostela, España. isabel.neira@usc.es

Maricruz Lacalle-Calderón es Profesora Doctora del Departamento de Economía Aplicada en la Facultad de Ciencias Económicas y Empresariales de la Universidad Autónoma de Madrid, España. maicu.lacalle@ uam.es

Marta Portela es Profesora de Economía Financiera y Contabilidad en la Facultad de Administración y Dirección de Empresas de la Universidad de Santiago de Compostela, España. marta.portela@usc.es 


\section{I}

\section{Introducción}

Si bien la eficacia de la asistencia oficial para el desarrollo (AOD) ha sido objeto de numerosos estudios empíricos, su efecto esperado en el crecimiento de los países receptores continúa siendo motivo de debate (Easterly, 2008; Gibson y otros, 2009).

En varios de esos estudios se analizan también otros factores que pueden incidir en la eficacia de la AOD, como las instituciones o las relaciones sociales, y que podrían promover u obstaculizar indirectamente el proceso de crecimiento. Por ejemplo, de acuerdo con Burnside y Dollar (2000), la posibilidad de que la AOD tenga un efecto positivo en el crecimiento depende de la existencia de políticas comerciales, monetarias y fiscales sólidas. Kaufmann, Kraay y Zoido-Lobatón (1999) proponen seis indicadores de gobernanza que según ellos pueden funcionar como importantes criterios de selección para la AOD. Rivera-Batiz (2002) señala que la democracia que mejora la gobernanza aumenta el crecimiento al reducir la corrupción. Choritz (2002); Simon y McGillivray (2003), y Knack (2001) subrayan la necesidad de conocer y comprender el capital social y otros factores impulsores del crecimiento existentes en la región, antes de la implementación de políticas y proyectos de desarrollo. Baliamoune-Lutz y Mavrotas (2009) investigan si el capital social y las instituciones incrementan la eficacia de la AOD. Con excepción de este último trabajo, que presenta algunas limitaciones, no se encuentra en la literatura ninguna otra prueba empírica con respecto al efecto macroeconómico del capital social en la eficacia de la AOD ${ }^{1}$.

La aplicación del modelo básico de dos brechas (Chenery y Strout, 1966) indica que la AOD es necesaria para cerrar la diferencia entre ahorro e inversión en los países pobres. Por lo tanto, cuando la asistencia se utiliza para financiar la inversión productiva, se puede esperar que tenga un efecto positivo en el crecimiento. Sin embargo, la decisión de utilizar la AOD para la inversión productiva, y su consiguiente efecto en el crecimiento, pueden estar influenciados por los niveles o la calidad del capital social de un determinado país.

Siguiendo a Burnside y Dollar (2000) y BaliamouneLutz y Mavrotas (2009), en este estudio se investiga

1 Si bien otros autores, como Knowles (2007), han estudiado el efecto del capital social en la asignación de la asistencia, esta área de investigación excede el objetivo de este trabajo. si el efecto de la AOD en el crecimiento depende del ambiente social e institucional del país receptor, que puede medirse - y se ha medido- sobre la base de variables como la buena gobernanza, la democracia o el capital social. Se procura probar la hipótesis de que el efecto de la AOD en el crecimiento en América Latina depende de las reservas existentes de capital social, y se utiliza la "confianza" como sustituto más preciso de esa variable ${ }^{2}$. Para ello se emplea un procedimiento de estimación superior al mínimo cuadrado ordinario (MCO). De este modo, el presente estudio contribuye a la literatura al examinar la interacción entre AOD y capital social (medido por la confianza) como determinante del crecimiento del producto interno bruto (PIB).

Mediante un modelo de crecimiento neoclásico modificado, se estudia empíricamente la eficacia de la AOD en presencia de confianza a partir del análisis de un panel incompleto de 18 países latinoamericanos en el período 2001-2010 ${ }^{3}$. Los resultados muestran que la AOD tiene un efecto positivo y estadísticamente significativo en el crecimiento en un ambiente con un alto nivel de confianza, pero ningún efecto en su ausencia.

El artículo se divide en seis secciones, incluida esta Introducción. En la segunda sección se resumen los trabajos más importantes de la literatura sobre la eficacia de la AOD como motor del crecimiento. En la tercera se analiza la confianza como forma de capital social y se procura determinar la manera en que esta puede condicionar la eficacia de la AOD. En la cuarta sección se presentan los datos y el modelo de datos de panel utilizado para examinar la dinámica de la AOD, la confianza y el efecto de su interacción en el crecimiento económico. En la quinta sección se discuten los resultados de la comparación de la eficacia de la AOD en presencia y ausencia de confianza, y en la sexta y última se presentan las conclusiones.

\footnotetext{
2 De acuerdo con la literatura reciente, la confianza es probablemente la variable sustitutiva más precisa para medir el capital social (Knack y Keefer, 1997; Whiteley, 2000; Beugelsdijk y Van Schaik, 2005; Helliwell y Putnam, 2000; Temple, 2001; Dollar y Kraay, 2002; Neira, Vázquez y Portela, 2009).

3 Específicamente: Argentina, Bolivia (Estado Plurinacional de), Brasil, Chile, Colombia, Costa Rica, Ecuador, El Salvador, Guatemala, Honduras, México, Nicaragua, Panamá, Paraguay, Perú, República Dominicana, Uruguay y Venezuela (República Bolivariana de).
} 


\section{II}

\section{Asistencia oficial para el desarrollo y crecimiento económico}

En varios estudios empíricos recientes se ha procurado determinar si la AOD tiene o no un efecto macroeconómico en el crecimiento. Aunque la evidencia es todavía ambigua y el debate continúa, se pueden definir dos posiciones principales, pero opuestas, sobre la eficacia de la asistencia (Easterly, 2008; Sachs, 2011).

En el período posterior a la Segunda Guerra Mundial, la AOD surgió como un imperativo, pues se necesitaban flujos financieros externos para reducir las brechas internas y externas que existían en las economías de los países pobres (Chenery y Strout, 1966). En las décadas siguientes, se realizó un esfuerzo de investigación decidido a demostrar la eficacia de la AOD como motor del crecimiento. En algunos estudios se obtuvieron resultados positivos (Levy, 1988), pero en otros estos fueron claramente negativos (Mosley, Hudson y Horrel, 1987; Mosley, 1980; Boone, 1996). En muchos de esos estudios no se abordó el carácter endógeno de la AOD, lo que puede explicar sus resultados contradictorios (Arndt, Jones y Tarp, 2010). En un estudio realizado en 2000, Burnside y Dollar (2000) hallaron que la AOD "funciona" en "buenos marcos normativos". La creencia de que la AOD estimula el crecimiento económico, reduce la pobreza y mejora los indicadores sociales dentro de buenos marcos normativos condujo al Banco Mundial a incrementar sus presupuestos de AOD en todo el mundo (Easterly, 2003).

Desde la divulgación del trabajo de Burnside y Dollar (2000), en varios estudios se han producido resultados opuestos. Hansen y Tarp (2000 y 2001); Dalgaard y Hansen (2001); Lensink y White (2001), y
Clemens y otros (2012) encuentran una relación causal positiva entre la asistencia y el crecimiento económico, que no depende de un buen marco normativo o de instituciones de calidad, pues sus resultados indican que la AOD fomenta el crecimiento incluso en países con un marco normativo desfavorable. Por el contrario, en varios otros estudios se halló que la AOD no tiene efectos en el crecimiento, haya un marco normativo sólido o no (Easterly, 2003; Easterly, Levine y Roodman, 2004; Rajan y Subramanian, 2008). Estos autores afirman que ningún tipo de asistencia (a corto plazo u otra) tiene efectos positivos en el desarrollo. Además, tampoco encuentran diferencias entre los efectos en el crecimiento de la asistencia bilateral y de la asistencia multilateral. Djankov, Montalvo y Reynal-Querol (2006 y 2008) sostienen que la AOD repercute negativamente en la democracia y en el crecimiento económico en los países en desarrollo. Los principales problemas que enfrentaron estos autores al intentar estimar el efecto de la AOD en el crecimiento fueron la naturaleza errática de dicha asistencia, la escasa calidad de los datos, la baja proporción de AOD con respecto al PIB en la mayoría de los países receptores, problemas de endogeneidad y el uso de instrumentos débiles (Tarp, 2006).

Dos meta-análisis recientes ofrecen resultados contrastantes. Mientras que Doucouliagos y Paldam (2011) hallan que la AOD es ineficaz para promover el crecimiento, Mekasha y Tarp (2013) encuentran que su efecto en el crecimiento es positivo y estadísticamente significativo. Una amplia revisión de la literatura demuestra que el debate continúa.

\section{III}

\section{Capital social, asistencia oficial para el desarrollo y crecimiento económico}

El capital social no es homogéneo: su naturaleza y las formas que adopta cambian con el tiempo según el tipo de equilibrio que existe entre las organizaciones públicas y privadas y la situación en el país de que se trate. Hay muchas definiciones diferentes de capital social, pues el concepto es relevante para numerosas disciplinas, como 
la sociología, las ciencias políticas y la economía, entre otras. La mayoría de esas definiciones utilizan términos como "redes", "confianza" y "reglas" o "normas". Uno de los aforismos frecuentemente utilizados para describir el capital social es "no es lo que sabes, sino a quién conoces". De acuerdo con Coleman (1988 y 2000), el capital social es algo que facilita la acción individual o colectiva y se genera mediante redes de relaciones, reciprocidad, confianza y normas sociales. Esta definición de capital social es similar a las de Bourdieu (1986) y Loury (1977). Para Coleman (1988), el capital social puede describirse como un recurso neutral derivado de la estructura de la sociedad que facilita ciertas acciones por parte de los individuos o actores colectivos, permitiendo de ese modo el logro de determinados fines que, en ausencia de capital social, no podrían alcanzarse.

Desde un punto de vista político, Putnam (1995) describe el capital social como elementos de organización social, entre ellos, redes, normas y confianza que facilitan la coordinación y la cooperación para el beneficio mutuo. Las definiciones propuestas por la Organización para la Cooperación y el Desarrollo Económicos (OCDE) y el Banco Mundial son similares. De acuerdo con la primera, el capital social incluye redes, normas, valores y acuerdos que facilitan la cooperación dentro de los grupos o entre ellos (OCDE, 2001). A su vez, el Banco Mundial define el capital social como las instituciones, relaciones y normas que conforman la calidad y la cantidad de interacciones sociales de una sociedad (Banco Mundial, 1985). Por lo tanto, la expresión de una relación, de un vínculo entre la confianza y la cooperación cívica es inherente a la idea de capital social. Dicho capital es el resultado de un proceso de interacción dinámica que puede aumentarse o destruirse, ya sea de manera deliberada o no, y requiere una inversión continua. Así, por todas esas razones, el capital social debería considerarse simplemente como tal: una forma de capital. Los principales aportes a esta corriente de opinión se resumen en el cuadro 1.

CUADRO 1

Definiciones de capital social

\begin{tabular}{lll}
\hline Autor & Elementos generadores & Consecuencias \\
\hline Bourdieu (1985) & Redes permanentes y afiliación a un grupo & $\begin{array}{l}\text { Asegurar a los miembros un conjunto de } \\
\text { recursos actuales o potenciales }\end{array}$ \\
\hline Coleman (1988) & Aspectos de la estructura social & $\begin{array}{l}\text { Facilitar determinadas acciones comunes } \\
\text { por parte de agentes dentro de la estructura }\end{array}$ \\
\hline Putnam, Leonardi y Nanetti (1993) & $\begin{array}{l}\text { Aspectos de organizaciones sociales como } \\
\text { redes, normas y confianza }\end{array}$ & $\begin{array}{l}\text { Facilitar la acción y la cooperación para el } \\
\text { beneficio mutuo }\end{array}$ \\
\hline Banco Mundial (1998) & Instituciones, relaciones y normas & $\begin{array}{l}\text { Conformar la calidad y la cantidad de } \\
\text { interacciones sociales de una sociedad }\end{array}$ \\
\hline ocDE (2001) & $\begin{array}{l}\text { Redes, normas, valores y opiniones } \\
\text { compartidas }\end{array}$ & $\begin{array}{l}\text { Facilitar la cooperación dentro de los } \\
\text { grupos y entre estos }\end{array}$ \\
\hline CEPAL (2002) & $\begin{array}{l}\text { El capital social es el conjunto de normas, } \\
\text { instituciones y organizaciones de una } \\
\text { sociedad }\end{array}$ & $\begin{array}{l}\text { Promover la confianza y la cooperación } \\
\text { entre las personas, las comunidades y la } \\
\text { sociedad en general }\end{array}$ \\
\hline Social Capital Interest Group (SCIG) (1999) & $\begin{array}{l}\text { Los potenciales beneficios, ventajas y } \\
\text { tratamiento preferencial que surgen como } \\
\text { resultado de la conmiseración y el sentido } \\
\text { de obligación que una persona siente } \\
\text { hacia un grupo o el grupo siente hacia una } \\
\text { persona }\end{array}$ & $\begin{array}{l}\text { Incluir los potenciales beneficios, ventajas } \\
\text { tratamiento preferencial que surgen de la } \\
\text { conmiseración de una persona y el sentido } \\
\text { de obligación hacia su yo idealizado }\end{array}$ \\
\hline
\end{tabular}

Fuente: I. Neira, E. Vázquez y M. Portela, "An empirical analysis of social capital and economic growth in Europe (1980-2000)", Social Indicators Research, vol. 92, $\mathrm{N}^{\circ}$ 1, Springer, 2009.

Nota: El Social Capital Interest Group (SCIG) está afiliado a la Universidad de Michigan.

En un intento de medir el capital social, en algunos estudios empíricos se han utilizado diferentes variables para tratar de captar este escurridizo concepto y sus múltiples dimensiones (Durlauf, 2002; Bjørnskov, 2006). Sin embargo, Oorschot y Arts (2005) señalan que hay un consenso cada vez mayor acerca de la posibilidad de agrupar los indicadores empíricos del capital social en tres dimensiones generales basadas en las definiciones anteriores. La primera corresponde a la confianza social, que es la variable utilizada con mayor frecuencia en la 
literatura empírica sobre capital social (Knack y Keefer, 1997; Whiteley, 2000; Beugelsdijk, De Groot y Van Schaik, 2004; Helliwell y Putnam, 2000; Temple, 2001; Dollar y Kraay, 2002). La confianza social generalmente supone diferentes tipos de confianza, que van de la existente en los miembros de la familia a aquella en los vecinos, la población del país y así sucesivamente. Esta primera dimensión es la variable que se ha elegido para medir el capital social en este trabajo. La segunda variable son las redes sociales, que con frecuencia se utiliza para medir la participación en diversas asociaciones en un intento por evaluar la integración social de los miembros de la comunidad analizada (Helliwell, 1996; Knack y Keefer, 1997; Beugelsdijk y Van Schaik, 2005; Hall, 1999). La tercera dimensión, normas sociales, se emplea para analizar valores cívicos y normas compartidas que se reflejan en variables como el nivel de corrupción, el grado de democracia, el índice de criminalidad, el divorcio y los niveles de desempleo, entre otras (Putnam, 2000; Bartolini y Bonatti, 2008).

Existen varios estudios en los que se ha procurado determinar si un mayor grado de capital social siempre se relaciona positivamente con el desarrollo económico y si las distintas etapas de desarrollo requieren diferentes combinaciones de capital social. También es posible que, en ciertos momentos o en determinadas sociedades, haya formas particulares de capital social antagonista. Algunos autores proponen la idea de que el capital social tiene un efecto negativo en la sociedad. El excesivo fervor religioso con que algunos países dirigen a sus sociedades puede redundar en guerras o desencadenar otra clase de conflictos, y la afiliación a grupos fervientes de ese tipo puede producir efectos negativos. Fukuyama (1999) afirma que tanto el Ku Klux Klan como la Mafia alcanzan fines cooperativos sobre la base de normas compartidas $y$, en consecuencia, tienen capital social, pero también producen numerosas externalidades negativas para la sociedad más grande en la que están incorporados. No obstante, aunque algunas redes pueden tener un impacto negativo si se concentran en sí mismas en detrimento de la sociedad en su conjunto, generalmente se asume que las relaciones de confianza son factores positivos en términos de cohesión social y éxito económico (Beugelsdijk, De Groot y Van Schaik, 2004; Beugelsdijk y Van Schaik, 2005; Helliwell y Putnam, 2000; Neira, Vázquez y Portela, 2009). La presencia de capital social (medido por la confianza) dentro de las estructuras sociales puede mejorar muchas actividades y disminuir su costo, promoviendo de ese modo el proceso de desarrollo. Estas actividades incluyen la toma de decisiones colectiva, la coordinación de diferentes iniciativas, la difusión de la innovación, entre otras. En presencia de capital social, esas actividades se realizan de manera más eficiente porque, en un contexto caracterizado por la confianza y la búsqueda de objetivos comunes, las personas están más dispuestas a cooperar unas con otras (Knack y Keefer, 1997; Fukuyama, 2001; Adler y Kwon, 2002).

Sin embargo, no existe en la literatura ningún estudio empírico en que se examine la manera en que la confianza, como forma de capital social, puede incrementar la eficacia de la AOD como motor del crecimiento ${ }^{4}$. Desde la perspectiva de los países que reciben AOD, se pueden postular tres maneras básicas en que la confianza puede modificar la eficacia de la asistencia y, en consecuencia, influir en las trayectorias de crecimiento económico de esos países.

En primer lugar, la confianza reduce los costos de transacción y facilita el flujo de información. Debido a que el sistema de ayuda externa está extremadamente fragmentado (Easterly, 2008), un ambiente donde predomine la confianza en el país receptor favorecerá la coordinación dentro del sistema, la colaboración entre los agentes, el consenso sobre objetivos globales y específicos, y la comunicación. Todos esos elementos ayudarán a garantizar que los escasos recursos se utilicen donde más se necesitan. Una burocracia transparente y la existencia de mecanismos para promover el diálogo y resolver los conflictos son condiciones necesarias para el uso exitoso de la AOD. Por el contrario, en ausencia de confianza, los agentes probablemente trabajarán para alcanzar metas contradictorias o duplicarán objetivos y tareas.

En segundo lugar, la confianza reduce el comportamiento oportunista. En contextos caracterizados por bajos niveles de confianza existe el riesgo de que la cooperación beneficie a los miembros más ricos o egoístas de las sociedades receptoras, que no tienen consideración con respecto a sus compatriotas. Un ambiente en que predomine la confianza es crucial para que la AOD se canalice en inversiones productivas en beneficio de las poblaciones más vulnerables. La confianza entre los países donantes y receptores y entre los gobiernos receptores y la sociedad civil es de vital importancia, porque el uso adecuado de la AOD necesita la combinación de diferentes formas de conocimiento, que están incorporadas en todos los diversos tipos de agentes sociales.

\footnotetext{
4 A nuestro entender, solo Baliamoune-Lutz y Mavrotas (2009) han realizado un estudio de este tipo, pero no utilizaron la confianza como variable sustitutiva del capital social. En su lugar, emplearon el fraccionamiento etnolingüístico como variable sustitutiva de la cohesión social y la cohesión social como principal indicador de capital social.
} 
En tercer lugar, la confianza sienta las bases para un sentido de pertenencia por parte de los receptores, sin la cual esos países no asumirán los compromisos necesarios para asegurar el progreso ideal de los proyectos de desarrollo (Gibson y otros, 2009). Dichos proyectos deberían ir acompañados de la propiedad local de los fondos correspondientes. Knack (2001) y Dollar y Pritchett (1998) hacen hincapié en el papel de la participación de los ciudadanos y el capital social en los países receptores, para asegurar que el sistema de ayuda externa sea eficaz. Por todas estas razones, se puede asumir que la confianza tiene un efecto positivo en la eficacia de la AOD como motor del crecimiento.

La literatura sobre la eficacia de la AOD en la promoción del crecimiento incluye varios estudios en los que se abordan factores distintos del capital social relacionados con la calidad de la estructura institucional del país receptor. Esos factores comprenden buena gobernanza, buenas políticas y un ambiente democrático. Dollar y Pritchett (1998) afirman que la asistencia para el desarrollo fomenta el crecimiento económico y ayuda a reducir la pobreza si los gobiernos locales son buenos administradores de sus instituciones sociales, políticas y económicas. Burnside y Dollar (2000) señalan que, para que la AOD sea eficaz, se necesitan mejores prácticas de formulación de políticas fiscales, monetarias y comerciales en los países receptores. Kaufmann, Kraay y Zoido-Lobatón (1999) proponen seis indicadores de buena gobernanza que, según ellos, pueden funcionar como importantes criterios de selección para la asistencia para el desarrollo. Además, Rivera-Batiz (2002) sostiene que la democracia que mejora la gobernanza aumenta el crecimiento al reducir la corrupción.

La premisa de las "buenas políticas" ha configurado las políticas de AOD desarrolladas en la última década. Sin embargo, autores como Baliamoune-Lutz y Mavrotas (2009) sostienen que estas no son un factor determinante clave y que factores como el capital social son más relevantes para determinar si la AOD se utiliza de manera satisfactoria o no. En esa misma línea, Choritz (2002), Simon y McGillivray (2003), Knack (2001) y Baliamoune-Lutz y Mavrotas (2009) subrayan la necesidad de una mejor comprensión del capital social existente en una región receptora, antes de que las autoridades comiencen a formular políticas o diseñar proyectos de desarrollo. En otras palabras, es preciso identificar con anticipación la reserva de capital social existente en la región receptora, porque esta puede acrecentar o disminuir la eficacia de la AOD en la promoción del proceso de crecimiento.

\section{Análisis empírico}

Los estudios sobre los efectos de la AOD en el crecimiento se basan generalmente en una muestra de países de todo el mundo y utilizan variables ficticias específicas para los distintos continentes. La muestra de 18 países latinoamericanos de este trabajo se emplea para estudiar el papel de la confianza en la determinación de la eficacia de la AOD en esa área geográfica. En general, los datos fiables sobre la influencia ejercida por el capital social en los países receptores de AOD son bastante escasos. Sin embargo, en el caso de América Latina, que es una importante región receptora de AOD, la base de datos de Latinobarómetro proporciona muy buenos datos sobre la confianza, que es la variable sustitutiva del capital social utilizada en este estudio.

\section{Datos}

El conjunto de datos empleado para analizar los efectos de la confianza en la eficacia de la AOD se obtuvo al combinar varias fuentes (véase el cuadro A.1 del anexo 1). Los registros de las variables se agruparon por país y año. Después de excluir algunos registros que no pudieron agruparse, se obtuvo un conjunto de datos final compuesto por un panel incompleto de 18 países en el período 2001-2010.

La variable dependiente es el crecimiento económico ${ }^{5}$, medido por el logaritmo del PIB real a paridad del poder adquisitivo $\left(L_{o g} G D P_{i t}\right)$ tomado de los Indicadores del Desarrollo Mundial (Banco Mundial, 2014). Las principales variables explicativas son la AOD (Log_ODA), medida como el logaritmo de la AOD neta

\footnotetext{
5 Puesto que se utiliza el método generalizado de momentos (MGM), la variable dependiente estimada es el crecimiento económico. El MGM utiliza variables en diferencias (véase la ecuación (2)) pero, dado que las variables están en forma logarítmica, sus diferencias se convierten en tasas de crecimiento para las variables originales en niveles (Acemoglu y otros, 2008).
} 
total (OCDE, 2013), y la confiANZA (trust), medida como el porcentaje de personas que respondieron "se puede confiar en la mayoría de las personas" a la pregunta "en general, ¿diría usted que se puede confiar en la mayoría de las personas o que nunca se es lo suficientemente cuidadoso al tratar con los demás?" (Latinobarómetro, 2010). Otras variables utilizadas en la literatura como variables explicativas del crecimiento y que se incluyen en el modelo de este trabajo son: población (Log_popu), medida por el logaritmo del número total de personas en el país; empleo (employment), medido por la proporción de la población total de un país (de 15 años de edad o más) que tiene un empleo; e inversión ( $\left.\log \_g c f\right)$, que se mide por el logaritmo de la formación bruta de capital. Las cifras correspondientes a estas tres variables se tomaron de la base de datos de los Indicadores del Desarrollo Mundial (Banco Mundial, 2014). La variable educación $(e d u)$, medida por el total de años de instrucción, se tomó de Barro y Lee (2012). De acuerdo con Acemoglu y otros (2008) y Burnside y Dollar (2000), también se introdujo el nivel de democracia (democ) del país como variable de control (tomado de Polity IV, 2013), pues se considera que la democracia es una variable separada, independiente del capital social.

En el mapa 1 y el gráfico 1 se ilustran los niveles de AOD neta recibida por cada país durante el período de análisis. En los mapas 2 y 3 se muestran los niveles medios de confianza y democracia en cada país en los años seleccionados. Debido a que la cantidad neta de AOD recibida por cada país cambia considerablemente de un año a otro dentro del período de estudio, se representaron esos datos en el mapa 1 y el gráfico 1 . En el caso de la confianza y la democracia, al tratarse de datos muy estables entre países y dentro de ellos, simplemente se incluyeron los valores medios relativos al período 2001-2010.

MAPA 1

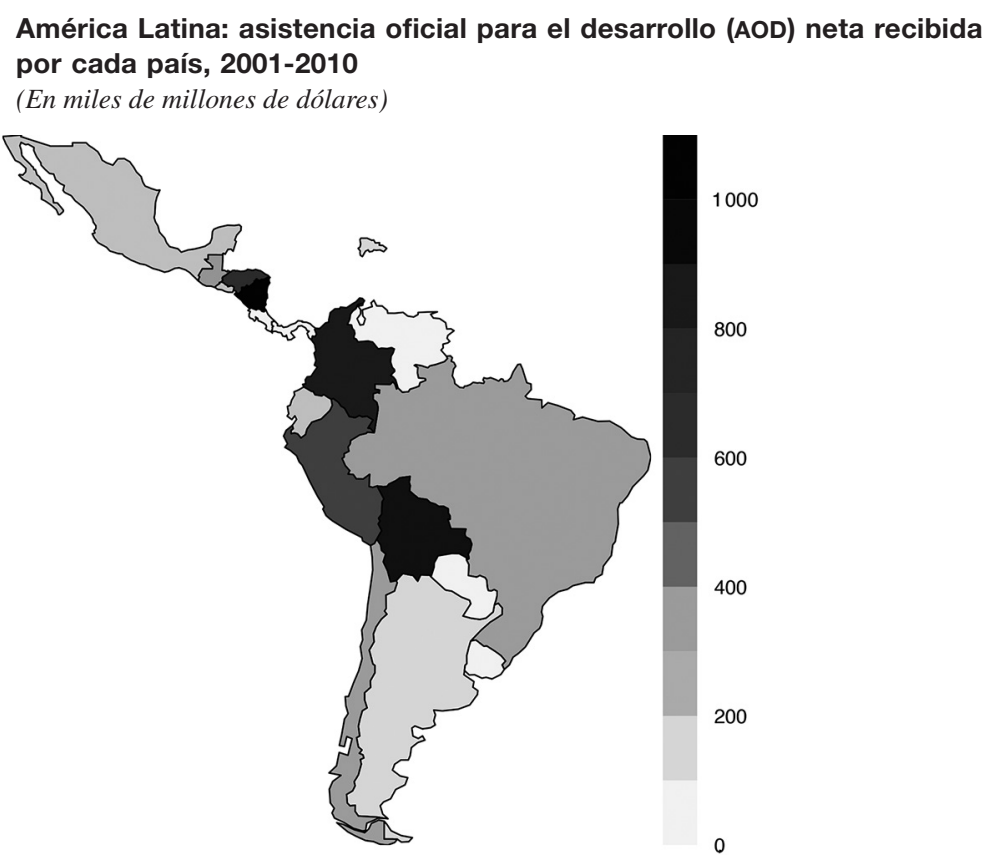

Fuente: Elaboración propia sobre la base de datos de la Organización para la Cooperación y el Desarrollo Económicos (OCDE) (promedio en años seleccionados).

Nota: Los límites que figuran en este mapa no implican su apoyo o aceptación oficial por las Naciones Unidas.

Todos los países de la muestra son receptores de AOD y, como puede observarse en el mapa 1 y el gráfico 1, Haití y Nicaragua son los principales receptores que registraron importantes cambios en los volúmenes de AOD recibidos de un año a otro. Entre otros países que reciben grandes cantidades de AOD se encuentran Bolivia (Estado Plurinacional de), Honduras y Colombia. Por otra parte, Venezuela (República Bolivariana de), el Uruguay, Costa Rica y el Paraguay recibieron los volúmenes más pequeños de AOD neta. 
MAPA 2

América Latina: niveles de confianza en cada país ${ }^{a}$, promedio en años seleccionados entre 2001 y 2010 (En porcentajes)

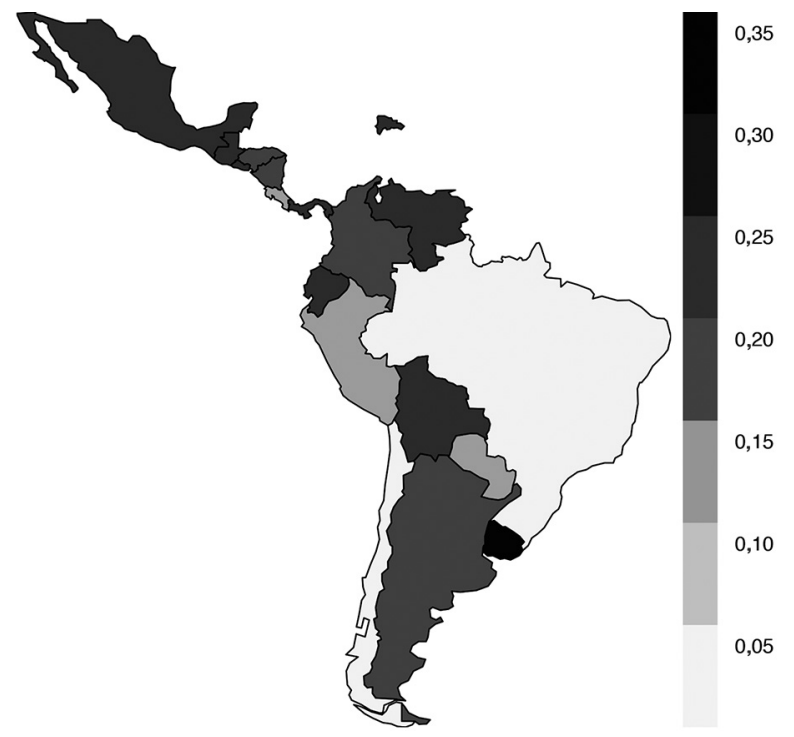

Fuente: Elaboración propia sobre la base de datos de Latinobarómetro (2010).

Nota: Los límites que figuran en este mapa no implican su apoyo o aceptación oficial por las Naciones Unidas.

a Porcentaje de personas que respondieron "se puede confiar en la mayoría de las personas" a la pregunta "en general, ¿diría usted que se puede confiar en la mayoría de las personas o que nunca se es lo suficientemente cuidadoso al tratar con los demás?".

MAPA 3

América Latina: niveles de democracia en cada país, promedio en años seleccionados entre 2001 y 2010 (Índice)

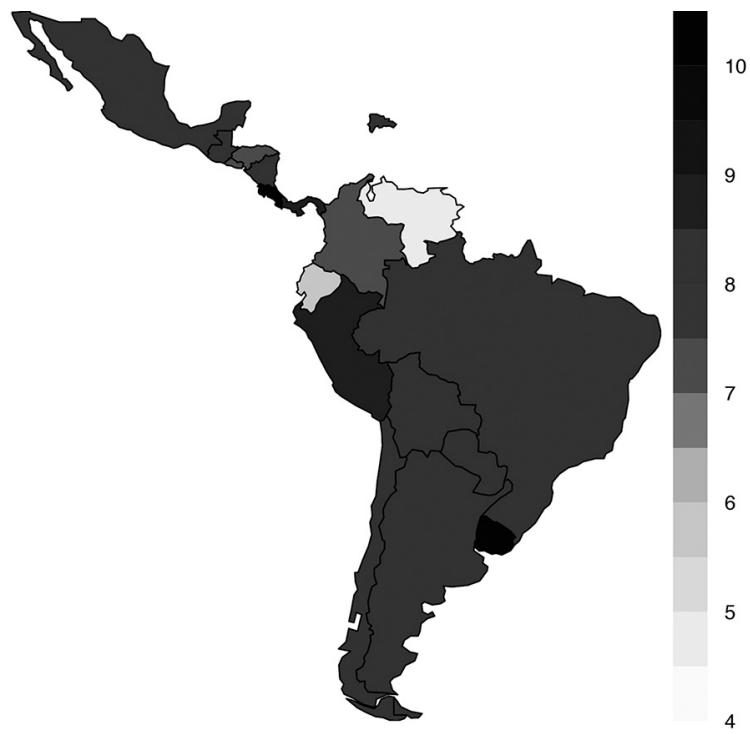

Fuente: Elaboración propia sobre la base de datos del proyecto Polity IV (2013).

Nota: Los límites que figuran en este mapa no implican su apoyo o aceptación oficial por las Naciones Unidas. 
GRÁFICO 1

América Latina: asistencia oficial para el desarrollo (AOD) neta recibida por cada país, 1996-2011

(En miles de millones de dólares)

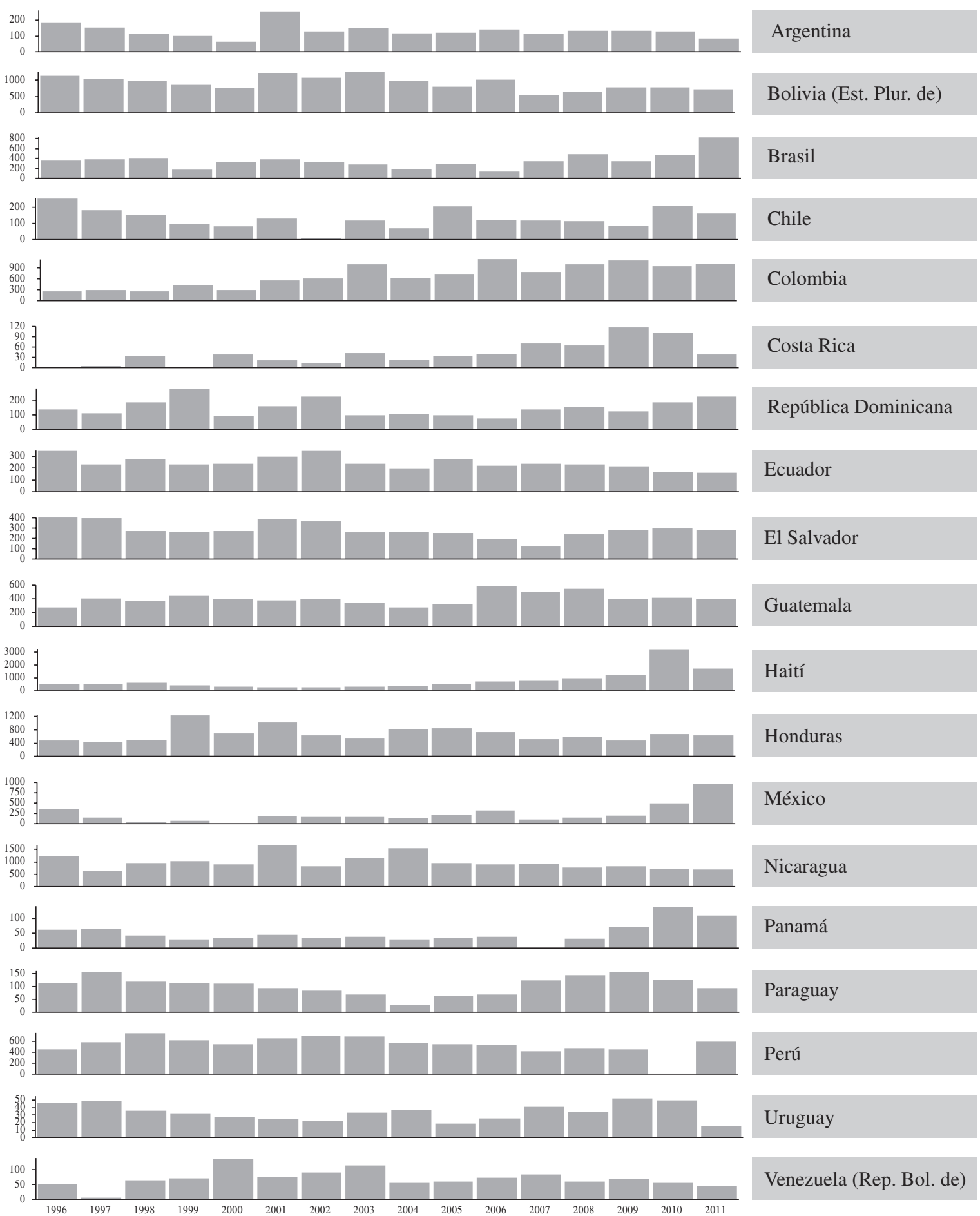

Fuente: Elaboración propia sobre la base de datos de la Organización para la Cooperación y el Desarrollo Económicos (OCDE). 
Los niveles de confianza son bajos en todos los países de la muestra. Solo entre el $18 \%$ y el $32 \%$ de la población de esos países respondió que "se puede confiar en la mayoría de las personas" a la pregunta "en general, ¿diría usted que se puede confiar en la mayoría de las personas o que nunca se es lo suficientemente cuidadoso al tratar con los demás?". Los países con los valores más altos son la República Dominicana, el Uruguay, México y Guatemala. Los países con los valores más bajos son Venezuela (República Bolivariana de), la Argentina, Honduras y Bolivia (Estado Plurinacional de).

Los datos relativos a la democracia toman valores enteros que van de 1 (falta total de democracia) a 10 (el mayor nivel de democracia). En este caso, el Ecuador, Venezuela (República Bolivariana de), Haití y Honduras son los países con los coeficientes más altos, mientras que la Argentina, Guatemala, el Brasil y la República Dominicana presentan los más bajos. En consecuencia, la correlación entre los datos sobre confianza y democracia es baja. En los países donde los niveles de confianza son altos, el nivel de democracia puede ser bajo, medio o alto.

En el cuadro A.2. del anexo 1 se presentan las estadísticas descriptivas de todas las variables de las regresiones. En cada caso, se detallan los promedios y las desviaciones estándar, junto con el número total de países sobre los cuales se disponía de datos y el número total de observaciones. En el cuadro A.3. del anexo 1 se presentan las correlaciones entre las diferentes variables.

\section{Procedimiento de estimación}

El siguiente modelo analítico, que es un modelo neoclásico modificado, constituyó la base de este trabajo:

$$
y_{i t}=\alpha+\lambda y_{i t-1}+\beta_{i} x_{i t}^{\prime}+\delta_{i}+\omega_{t}+\varepsilon_{i t}
$$

donde el valor rezagado del log del PIB $\left(\log _{-} G D P_{i, t-1}\right)$ se utiliza para controlar la persistencia del crecimiento económico en el tiempo, mientras que $X^{\prime}{ }_{i t}$ incluye AOD y confianza, las principales variables independientes. También se incluye un grupo de variables de control en los principales modelos de crecimiento (educación, población, empleo, inversión y democracia). Además, $X^{\prime}{ }_{i t}$ contiene el término de interacción "Log_ODA*trust" para contrastar la hipótesis de que la eficacia de la AOD depende del nivel de capital social del país. También comprende el término de interacción " $L o g \_O D A * d e m o c$ " para comparar las diferentes medidas sociales e institucionales. Los términos $\delta_{i}$ y $\omega_{t}$ captan la heterogeneidad del país y tiempo no observable, pues se tiene en cuenta la presencia de efectos de país; $\alpha$ y $\beta$ son los parámetros que han de estimarse; y $\varepsilon_{i t}$ es el término de error, que capta todos los otros factores omitidos, y $\mathrm{E}\left[\varepsilon_{i t}\right]=0$ para i y t.

Siguiendo el procedimiento utilizado por Asiedu y Nandwa (2007), se estima la ecuación sobre la base del trabajo de Hansen y Tarp (2001), quienes analizaron varias regresiones de AOD para subrayar que las variables que la determinan no pueden ser exógenas a las regresiones de crecimiento. Esto significa que se necesitan alternativas a las estimaciones de MCO. La ecuación (2) es la especificación de un modelo de datos de panel dinámico del logaritmo del PIB real $\left(\log _{-} G D P_{i t}\right)$,

$$
\Delta y_{i t}=\alpha+\lambda \Delta y_{i t-1}+\beta_{i} \Delta x_{i t}^{\prime}+\Delta \varepsilon_{i t}
$$

Se estima la ecuación (2), un modelo de datos de panel dinámico, utilizando el método generalizado de momentos (MGM) propuesto por Arellano y Bond (1991). Este método tiene en cuenta efectos específicos de país no observados, errores de medición y problemas de endogeneidad, no solo en el caso de la variable dependiente rezagada, sino también en el de cualquier otro regresor (Arndt, Jones y Tarp, 2010). La principal razón teórica para utilizar el panel dinámico es que este modela un enfoque basado en el ajuste parcial. Si se trata de un proceso de ajuste parcial, el coeficiente de la variable dependiente rezagada mide la velocidad del ajuste. Además, dicha variable puede eliminar cualquier autocorrelación.

Para tratar la endogeneidad, el estimador MGM en diferencias de Arellano y Bond (1991) utiliza los niveles rezagados de la primera diferencia de las variables como instrumentos tanto para la variable dependiente rezagada como para las otras variables explicativas. Este método identifica el número de rezagos de la variable dependiente, las variables predeterminadas y las variables endógenas como instrumentos válidos, y muestra cómo combinar esos niveles rezagados con las primeras diferencias de las variables estrictamente exógenas en una matriz de instrumentos potencialmente grande. Se emplea el denominado estimador MGM en diferencias en dos etapas, que tiene en cuenta la heteroscedasticidad en los términos de error. El modelo de Arellano y Bond asume que cuando $x_{i t}$ y $\varepsilon_{i t}$ no están correlacionadas en serie, esta hipótesis puede contrastarse utilizando las pruebas estadísticas $\mathrm{m} 2$ para analizar la correlación serial de segundo orden en los residuos de la primera diferencia. Las pruebas de especificación son aplicables en este contexto. Una de ellas es la prueba de Sargan para restricciones de sobreidentificación (Sargan, 1958). Los resultados de esta prueba indican que no es necesario considerar otros instrumentos. 


\section{V}

\section{Resultados}

Los resultados de la estimación de los modelos en las ecuaciones (1) y (2) se presentan en el cuadro 2. Se recordará que el objetivo de este trabajo es determinar si la eficacia de la AOD en la promoción del crecimiento depende del nivel de confianza que existe en el país de que se trate.

Las pruebas de autocorrelación serial de Arellano y Bond (1991) presentaron los resultados esperados en todos los casos, excepto en el modelo 1 , que se incluyó para probar la solidez de los resultados. Estos resultados de prueba son siempre significativos para una autocorrelación de primer orden $(t-1)$, pero no lo son para la autocorrelación de segundo orden. La prueba de especificación de Sargan siempre acepta la hipótesis nula de la validez de las restricciones de sobreidentificación, indicando que la especificación del modelo y de los instrumentos es buena.

Los resultados presentados en el cuadro 2 muestran estimaciones de coeficientes con los signos esperados. Los resultados son siempre significativos estadísticamente.

Los resultados presentados en el cuadro 2 reflejan cuatro hallazgos principales. En primer lugar, hay una relación negativa y significativa entre la AOD y el crecimiento económico, y este resultado es constante en todas las especificaciones. Incluso al controlar por la confianza (modelo 1), por el término de interacción "Log_ODA*trust" (modelo 2), por la democracia (modelo 4) o por el término de interacción " $L O g_{-} O D A *$ democ" (modelo 5), el efecto de la AOD en el crecimiento se mantiene negativo. Estos resultados son similares a los obtenidos por Boone (1996), Easterly (2003), Rajan y Subramanian (2008) y Djankov, Montalvo y ReynalQuerol (2006 y 2008), quienes hallaron que la AOD tiene un impacto negativo en el crecimiento económico en los países en desarrollo.

En segundo lugar, la confianza, como forma de capital social, es positiva para la eficacia de la AOD en la promoción del crecimiento. El modelo (1) en el cuadro 2 indica que la confianza, por sí misma, es un factor explicativo importante del crecimiento. Los resultados en las columnas 2 y 3 son interesantes. La AOD tiene un efecto positivo y estadísticamente significativo en el crecimiento en los ambientes con altos niveles de confianza. Sin embargo, si se omite la interacción entre la asistencia y la confianza, la AOD deja de tener un efecto positivo en el crecimiento. Es también interesante que el coeficiente estimado para la interacción de la confianza y la AOD se mantiene positivo incluso cuando la AOD se

CUADRO 2

Resultados de la estimación para una muestra de 18 países, 2001-2010

\begin{tabular}{|c|c|c|c|c|c|c|c|}
\hline \multirow{2}{*}{\multicolumn{2}{|c|}{$\begin{array}{l}\text { Variable dependiente: } \\
\text { Variables independientes: }\end{array}$}} & \multicolumn{6}{|c|}{$\log G D P$} \\
\hline & & (1) & (2) & (3) & (4) & (5) & (6) \\
\hline \multicolumn{2}{|l|}{$\log O D A$} & $-0,0064 * * *$ & $-0,0074 * * *$ & & $-0,0094 * * *$ & $-0,0095 * * *$ & \\
\hline \multicolumn{2}{|c|}{ Trust } & $0,0788 * * *$ & & & - $\quad$ & - & \\
\hline \multicolumn{2}{|c|}{ Variable dependientete rezagada } & $0,5409 * * *$ & $0,5109 * * *$ & $0,5263 * * *$ & $0,6539 * * *$ & $0,6572 * * *$ & $0,6691 * * *$ \\
\hline \multicolumn{2}{|c|}{$\log g c f$} & $0,1551 * * *$ & $0,1519 * * *$ & $0,1551 * * *$ & $0,1373 * * *$ & $0,1396 * * *$ & $0,1343 * * * *$ \\
\hline \multicolumn{2}{|l|}{ Employment } & $0,0043 * * *$ & $0,0055 * * *$ & $0,0044 * * *$ & $0,0047 * * *$ & $0,0044 * * *$ & $0,0052 * * *$ \\
\hline \multicolumn{2}{|l|}{$E d u$} & $0,0104 * * *$ & $0,0082 * * *$ & $0,0109 * * *$ & $0,0062 * * *$ & $0,0061 * * *$ & $0,0051 * * *$ \\
\hline \multicolumn{2}{|l|}{ Log рори } & $0,9224 * * *$ & $0,9458 * * *$ & $0,9190 * * *$ & $0,6623 * * *$ & $0,6828 * * *$ & $0,6212 * * *$ \\
\hline \multicolumn{2}{|l|}{ Log ODA *trust } & & $0,0141 * * *$ & $0,0089 * * *$ & & & \\
\hline \multicolumn{2}{|l|}{ Democ } & & & & $0,0002 * * *$ & & \\
\hline \multicolumn{2}{|l|}{$\log O D A^{*}$ democ } & & & & & $0,00003 * * *$ & $0,00003 * * *$ \\
\hline Arellano y Bond & Primer orden & $-2,2478^{*}$ & $-2,1647^{*}$ & $-2,9171$ & $-2,4269^{*}$ & $-2,3995^{*}$ & $-0,0179 * * *$ \\
\hline $\begin{array}{l}\text { Prueba de } \\
\text { autocorrelación }\end{array}$ & Segundo orden $r$ & $-1,8521 * *$ & $-1,7874 * *$ & $-1,1069 * * * *$ & $-1,5201 * * *$ & $-1,5387 * * *$ & $-0,0234 * * *$ \\
\hline \multicolumn{2}{|c|}{ Prueba de especificación de Sargan } & 0,9 & 0,9 & 0,9 & 0,9 & 0,9 & 0,9 \\
\hline \multicolumn{2}{|c|}{ Total de observaciones } & 218 & 218 & 218 & 256 & 256 & 256 \\
\hline
\end{tabular}

Fuente: Elaboración propia.

Nota: Todas las variables, excepto las variables de inflación y tasa de interés, están expresadas en forma logarítimica. *** Significativo al valor $\mathrm{p}<0,01$; ** significativo a $\mathrm{p}<0,5 ; *$ significativo a $\mathrm{p}<0,1$. La estadística-t Arellano y Bond significa $\rho=0$.

ODA: Asistencia oficial para el desarrollo (AOD). 
excluye de las regresiones. Todo esto significa que los efectos de la AOD en el crecimiento son sistemáticamente mayores en un ambiente con altos niveles de confianza que en uno donde estos son bajos. Tales resultados coinciden con los de Burnside y Dollar (2000) al mostrar que la eficacia de la AOD depende de otras variables (políticas fiscales, monetarias y comerciales) y, en este estudio, también del capital social. Asimismo son coherentes con las conclusiones de Baliamoune-Lutz y Mavrotas (2009), quienes hallaron que cuando la AOD interactúa con la cohesión social, el resultado es un coeficiente significativamente positivo.

En tercer lugar, conforme con resultados anteriores, los modelos 4 a 6 evidencian que la AOD tiene un efecto negativo significativo en el crecimiento, pero este resultado se vuelve positivo y significativo en los países con altos niveles de democracia. Específicamente, estos tres modelos muestran que la democracia por sí misma y el término de interacción " $L o g \_O D A * d e m o c$ " tienen coeficientes estimados positivos. Estos hallazgos son coherentes con los de Burnside y Dollar (2000) y Collier y Dollar (2002), pues el efecto de la AOD es positivo en "buenos marcos normativos".

En cuarto lugar, y no menos importante que los resultados anteriores, al comparar las columnas 2 y 3 con las columnas 5 y 6 se observa que el coeficiente estimado para la interacción entre confianza y AOD es mayor que

\section{VI}

\section{Conclusiones}

En este estudio se analizó la interacción entre la AOD, el capital social y el crecimiento económico a fin de investigar si la eficacia de la asistencia para el desarrollo depende del nivel de confianza (una forma de capital social) existente en un país receptor. De acuerdo con hallazgos similares documentados en la literatura, se determinó que dicho nivel condiciona la eficacia de la AOD en la promoción del crecimiento: la asistencia para el desarrollo tiene un efecto positivo en el crecimiento en aquellos países donde existe un nivel significativo de confianza y un efecto negativo cuando esta no está presente. Además, se halló que la confianza es un factor más importante que la democracia para incrementar la eficacia de la AOD. En otras palabras, un ambiente donde ella predomina tiene un mayor efecto en la eficacia de la AOD para impulsar el crecimiento que un ambiente con altos niveles de democracia. Por lo tanto, aquel estimado para la interacción entre democracia y AOD. Esto significa que un ambiente donde predomina la confianza tiene mayor efecto en la eficacia de la AOD para promover el crecimiento que un ambiente con altos niveles de democracia. Por lo tanto, las agencias donantes deberían dirigir sus esfuerzos a mejorar las capacidades de una mayor proporción de la población en lugar de simplemente tratar de reemplazar infraestructuras primitivas con inversiones modernas y técnicamente sofisticadas (Ostrom, 2000). Las probabilidades de que la AOD sea eficaz aumentan al involucrar a la comunidad local. Es importante que los donantes conozcan las reservas de capital social existentes en el país antes de desarrollar políticas o diseñar proyectos. La evaluación del capital social podría combinarse con la evaluación de la pobreza y las políticas sociales deberían orientarse a identificar instituciones, redes y relaciones sociales que contribuyen al crecimiento y la reducción de la pobreza o los obstaculizan (Grootaert, 1998).

Por último, se realizó un análisis de solidez para determinar si los resultados anteriores fueron impulsados solo por unos pocos países. Dichos resultados son similares a los presentados en el cuadro $2^{6}$.

\footnotetext{
${ }^{6}$ Los resultados de solidez están disponibles a solicitud.
}

es importante que las inversiones de AOD se dirijan a los sectores que favorecerán el desarrollo del capital social como un medio para escapar de la pobreza. También es necesario involucrar en forma más directa a las comunidades locales, a fin de alcanzar los niveles requeridos para que la AOD sea más eficaz.

La presencia de capital social, medido por la confianza, es importante para el crecimiento y, cuando se combina con la AOD, puede aumentar su eficacia. Esto ocurre probablemente porque la confianza facilita la cooperación entre los individuos al reducir los costos de transacción. Sin embargo, una investigación profunda de este aspecto va más allá del alcance del presente estudio y requeriría un examen de los mecanismos de transmisión involucrados en la interacción entre la existencia de confianza y el crecimiento basado en la AOD. 
ANEXO

CUADRO A.1

América Latina (18 países): especificación de la lista de variables, 2001-2010

\begin{tabular}{|c|c|c|c|}
\hline Variable & Descripción & Unidades & Fuente \\
\hline \multicolumn{4}{|c|}{ Variable dependiente: } \\
\hline $\log G D P$ & $\begin{array}{l}\text { Logaritmo del PIB real, paridad del poder } \\
\text { adquisitivo (a precios constantes de } 2005 \text { ) }\end{array}$ & $\begin{array}{l}\text { Dólares } \\
\text { internacionales }\end{array}$ & Indicadores del Desarrollo Mundial \\
\hline \multicolumn{4}{|c|}{ Variables explicativas: } \\
\hline \multicolumn{4}{|c|}{ a) Persistencia del crecimiento económico en el tiempo } \\
\hline $\log G D P_{i, t-}$ & $\begin{array}{l}\text { 1Logaritmo del PIB real, paridad del poder } \\
\text { adquisitivo en el año precedente (constante 2005) }\end{array}$ & $\begin{array}{l}\text { Dólares } \\
\text { internacionales }\end{array}$ & Indicadores del Desarrollo Mundial \\
\hline \multicolumn{4}{|c|}{ b) Variables principales } \\
\hline $\log O D A$ & Logaritmo de AOD neta total & $\begin{array}{l}\text { Dólares } \\
\text { internacionales }\end{array}$ & $\begin{array}{l}\text { lción para la Cooperación y el } \\
\text { lo Económicos (OCDE) (base de datos } \\
\text { ité de Asistencia al Desarrollo (CAD)) }\end{array}$ \\
\hline Trust & Confianza & $\begin{array}{l}\text { Porcentaje de } \\
\text { personas }\end{array}$ & rómetro \\
\hline \multicolumn{4}{|c|}{ c) Variables de control } \\
\hline$E d u$ & Total de años de instrucción & Años & Base de datos de Barro y Lee (2012) \\
\hline Employment & $\begin{array}{l}\text { Relación entre empleo y población ( } 15 \text { años } \\
\text { de edad o más) }\end{array}$ & Porcentaje de personas & Indicadores del Desarrollo Mundial \\
\hline $\log g c f$ & Logaritmo de formación bruta de capital & Dólares internacionales & Indicadores del Desarrollo Mundial \\
\hline Log рори & Logaritmo de población total & Número de personas & Indicadores del Desarrollo Mundial \\
\hline Democ & Índice de democracia & $0-10$ & Polity IV ${ }^{\mathrm{a}}$ \\
\hline
\end{tabular}

Fuente: Elaboración propia.

Nota: AOD: Asistencia oficial para el desarrollo.

a Polity IV Database es un proyecto de investigación sobre libertad en el mundo, pero también es una de las bases de datos más importantes donde se recoge la evolución de los niveles de libertad en el planeta.

CUADRO A.2

Estadísticas descriptivas

\begin{tabular}{|c|c|c|c|c|c|c|c|c|}
\hline & $\log G D P$ & $\log O D A$ & Trust & $E d u$ & Employment & $\log g c f$ & Log рори & Democ \\
\hline Media & 24,93 & 363,84 & 0,19 & 6,91 & 58,88 & 3,04 & 16,27 & 7,48 \\
\hline Mediana & 24,54 & 236,89 & 0,19 & 7,18 & 58,30 & 3,05 & 16,07 & 8,00 \\
\hline Máximo & 28,34 & 3231,14 & 0,44 & 9,74 & 73,10 & 3,73 & 19,11 & 10,00 \\
\hline Mínimo & 21,27 & 1,28 & 0,02 & 3,27 & 43,20 & 2,40 & 13,50 & $-88,00$ \\
\hline Desviación estándar & 1,61 & 376,97 & 0,08 & 1,59 & 58,88 & 3,04 & 1,25 & 6,23 \\
\hline $\mathrm{N}^{\mathrm{o}}$ de observaciones & 332 & 300 & 260 & 340 & 340 & 340 & 340 & 252 \\
\hline
\end{tabular}

Fuente: Elaboración propia.

CUADRO A.3

Matriz de correlaciones

\begin{tabular}{lcccccccc}
\hline & Log GDP & Log gcf & Employment & Log ODA & Log popu & edu & democ & trust \\
\hline Log GDP & 1,00 & $-0,08$ & $-0,11$ & $-0,00$ & 0,95 & 0,17 & $-0,07$ & $-0,18$ \\
Log gcf & $-0,08$ & 1,00 & $-0,08$ & 0,12 & $-0,07$ & $-0,09$ & $-0,04$ & $-0,04$ \\
employment & $-0,11$ & $-0,08$ & 1,00 & 0,35 & 0,03 & $-0,15$ & $-0,12$ & $-0,14$ \\
Log ODA & $-0,00$ & 0,12 & 0,35 & 1,00 & 0,24 & $-0,38$ & $-0,12$ & $-0,17$ \\
Log popu & 0,95 & $-0,07$ & 0,03 & 0,24 & 1,00 & $-0,03$ & $-0,11$ & $-0,25$ \\
Edu & 0,17 & $-0,09$ & $-0,15$ & $-0,38$ & $-0,03$ & 1,00 & 0,05 & 0,04 \\
Democ & $-0,07$ & $-0,04$ & $-0,12$ & $-0,12$ & $-0,11$ & 0,05 & 1,00 & 0,07 \\
Trust & $-0,18$ & $-0,04$ & $-0,14$ & $-0,17$ & $-0,25$ & 0,04 & 0,07 & 1,00 \\
\hline
\end{tabular}

Fuente: Elaboración propia. 


\section{Bibliografía}

Acemoglu, D. y otros (2008), "Income and democracy", American Economic Review, vol. 98, $\mathrm{N}^{\circ}$ 3, Nashville, Tennessee, American Economic Association.

Adler, P.S. y S.W. Kwon (2002), "Social capital: prospects for a new concept", Academy of Management Review, vol. 27, $\mathrm{N}^{\circ} 1$, Briarcliff Manor, Academy of Management.

Arellano, M. y S. Bond (1991), "Some tests of specification for panel data: Monte Carlo evidence and an application of employment equations", Review of Economic Studies, vol. 58, № 2, Oxford University Press.

Arndt, C., S. Jones y F. Tarp (2010), "Aid, growth and development have we come full circle?", Journal of Globalization and Development, vol. 1, $\mathrm{N}^{\circ}$ 2, De Gruyter.

Asiedu, E. y B. Nandwa (2007), "On the impact of foreign aid in education on growth: how relevant is the heterogeneity of aid flows and the heterogeneity of aid recipients?", Review of World Economics, vol. 143, $\mathrm{N}^{\circ} 4$, Springer.

Baliamoune-Lutz, M. y G. Mavrotas (2009), "Aid effectiveness: looking at the aid-social capital-growth nexus", Review of Development Economics, vol. 13, $\mathrm{N}^{\circ} 3$, Wiley.

Banco Mundial (2014), "World Development Indicators" [en línea] http://data.worldbank.org/data-catalog/world-developmentindicators.

(1998), "The initiative on defining, monitoring and measuring social capital", Social Capital Initiative Working Paper, $\mathrm{N}^{\circ} 1$ [en línea] http://siteresources.worldbank.org/ INTSOCIALCAPITAL/Resources/Social-Capital-InitiativeWorking-Paper-Series/SCI-WPS-01.pdf.

(1985), World Development Report 1985, Washington, D.C.

Barro, R.J. y J. Lee (2012), "Full dataset" [en línea] http://www. barrolee.com/data/full1.htm.

Bartolini, S. y L. Bonatti (2008), "Endogenous growth, decline in social capital and expansion of market activities", Journal of Economic Behavior \& Organization, vol. 67, $\mathrm{N}^{\circ} 3-4$, Amsterdam, Elsevier.

Beugelsdijk, S., H.L.F. de Groot y T. van Schaik (2004), "Trust and economic growth: a robustness analysis", Oxford Economic Papers, vol. 56, $\mathrm{N}^{\circ} 1$, Oxford University Press.

Beugelsdijk, S. y T. van Schaik (2005), "Social capital and growth in European regions: an empirical test", European Journal of Political Economy, vol. 21, $\mathrm{N}^{\circ}$ 2, Amsterdam, Elsevier.

Bjørnskov, C. (2006), "The multiple facets of social capital", European Journal of Political Economy, vol. 22, $\mathrm{N}^{\circ} 1$, Amsterdam, Elsevier.

Boone, P. (1996), "Politics and the effectiveness of foreign aid", European Economic Review, vol. 40, No 2, Amsterdam, Elsevier

Bourdieu, P. (1986), "The forms of capital", Handbook of Theory and Research for the Sociology of Education, J. Richardson (ed.), Nueva York, Greenwood.

(1985), "The social space and the genesis of groups", Theory and Society, vol. $14, \mathrm{~N}^{\circ} 6$, Springer.

Burnside, G. y D. Dollar (2000), "Aid, policies and growth", The American Economic Review, vol. 90, $\mathrm{N}^{\circ}$ 4, Nashville, Tennessee, American Economic Association.

CEPAL (Comisión Económica para América Latina y el Caribe) (2002), Panorama Social de América Latina 2001-2002 (LC/G.2183-P), Santiago.

Chenery, H. y A. Strout (1966), "Foreign assistance and economic development", American Economic Review, vol. 56, $\mathrm{N}^{\circ} 4$ Nashville, Tennessee, American Economic Association.

Choritz, S. (2002), Literature Review of Evaluative Evidence on the Three Drivers of Effective Development: Ownership, Policy and Capacity Development, Nueva York, Programa de las Naciones Unidas para el Desarrollo (PNUD).

Clemens, M.A. y otros (2012), "Counting chickens when they hatch: timing and the effects of aid on growth", The Economic Journal, vol. 122, $\mathrm{N}^{\circ}$ 561, Wiley.
Coleman, J. (2000), Foundations of Social Theory, Cambridge, Massachusetts, Belknap Press of Harvard University Press.

(1988), "Social capital in the creation of human capital", American Journal of Sociology, vol. 94, Chicago, University of Chicago Press.

Collier, P. y A. Dollar (2002), "Aid allocation and poverty reduction", European Economic Review, vol. 46, № 8, Amsterdam, Elsevier.

Dalgaard C-J. y H. Hansen (2001), "On aid, growth and good policies", Journal of Development Studies, vol. 37, $\mathrm{N}^{\circ} 6$, Taylor \& Francis.

Djankov, S., J.G. Montalvo y M. Reynal-Querol (2008), "The curse of aid", Journal Economic Growth, vol. 13, $\mathrm{N}^{\circ} 3$, Springer.

(2006), "Does foreign aid help?", CATO Journal, vol. 26, $\mathrm{N}^{\circ} 1$, Washington, D.C., CATO Institute.

Dollar, D. y A. Kraay (2002), "Growth is good for the poor", Journal of Economic Growth, vol. 7, $\mathrm{N}^{\circ} 3$, Springer.

Dollar, D. y L. Pritchett (1998), Assessing Aid-What Works, What Doesn't, and Why, Nueva York, Oxford University Press.

Doucouliagos, H. y M. Paldam (2011), "The ineffectiveness of development aid on growth: an update", European Journal of Political Economy, vol. 27, $\mathrm{N}^{\circ}$ 2, Amsterdam, Elsevier.

Durlauf, S.N. (2002), "On the empirics of social capital", The Economic Journal, vol. 112, N ${ }^{\circ} 483$, Royal Economic Society.

Easterly, W. (2008), Reinventing Foreign Aid, Cambridge, Massachusetts, The MIT Press.

(2003), "Can foreign aid buy growth?", Journal of Economic Perspectives, vol. 17, $\mathrm{N}^{\circ}$ 3, Nashville, Tennessee, American Economic Association.

Easterly, W., R. Levine y D. Roodman (2004), "Aid, policies and growth: comment", American Economic Review, vol. 94, №3, Nashville, Tennessee, American Economic Association.

Fukuyama, F. (2001), "Social capital, civil society and development", Third World Quarterly, vol. 22, $\mathrm{N}^{\circ}$ 120, Taylor \& Francis.

(1999), Social Capital and Civil Society, Universidad George Mason.

Gibson, C.C. y otros (2009), The Samaritan's Dilemma, Oxford, Oxford University Press.

Grootaert, C. (1998), "Social capital: the missing link?", Social Capital Initiative Working Paper, $\mathrm{N}^{\mathrm{o}} 3$, Washington, D.C., Banco Mundial.

Hall, P. (1999), "Social capital in Britain", British Journal of Politics, vol. 29, $\mathrm{N}^{\circ} 3$, Cambridge University Press.

Hansen, H. y F. Tarp (2001), "Aid and growth regressions", Journal of Development Economics, vol. 64, № 2, Amsterdam, Elsevier.

(2000), "Aid effectiveness disputed", Journal of International Development, vol. 12, $\mathrm{N}^{\circ} 3$, Wiley.

Helliwell, J.F. (1996), "Economic growth and social capital in Asia", NBER Working Paper, $\mathrm{N}^{\circ}$ 5470, Cambridge, Massachusetts, National Bureau of Economic Research.

Helliwell, J.F. y R.D. Putnam (2000), "Economic growth and social capital in Italy", Social Capital: A Multifaceted Perspective, P. Dasgupta e I. Serageldin (eds.), Washington, D.C., Banco Mundial.

Kaufmann, D., A. Kraay y P. Zoido-Lobatón (1999), “Aggregating governance indicators", Policy Research Working Paper, $\mathrm{N}^{\circ}$ 2195, Washington, D.C., Banco Mundial.

Knack, S. (2001), "Aid dependence and the quality of governance: cross-country empirical tests", Southern Economic Journal, vol. 68, $\mathrm{N}^{\circ} 2$, Southern Economic Association.

Knack, S. y P. Keefer (1997), "Does social capital have an economic payoff? A cross-country investigation", Quarterly Journal of Economics, vol. 112, $\mathrm{N}^{\circ} 4$, Oxford University Press.

Knowles, S. (2007), "Social capital, egalitarianism and foreign aid allocations", Journal of International Development, vol. 19, $\mathrm{N}^{\circ} 3$, Wiley.

Latinobarómetro (2010) [en línea] http://www.latinobarometro.org/ latContents.jsp.y. 
Lensink, R. y H. White (2001), "Are there negative returns to aid?", The Journal of Development Studies, vol. 37, $\mathrm{N}^{\circ}$ 6, Taylor \& Francis.

Levy, V. (1988), "Aid and growth in Sub-Saharan Africa: the recent experience", European Economic Review, vol. 32, $\mathrm{N}^{\circ}$ 9, Amsterdam, Elsevier.

Loury, G.C. (1977), "A dynamic theory of racial income difference", Women, Minorities and Employment Discrimination, P.A. Wallace y A.M. LaMond (eds.), Lexington, Lexington Books.

Mekasha, T.J. y F. Tarp (2013), "Aid and growth: what meta-analysis reveals", The Journal of Development Studies, vol. 49, $\mathrm{N}^{\circ} 4$, Taylor \& Francis

Mosley, P. (1980), "Aid, savings and growth revisited", Oxford Bulletin of Economics and Statistics, vol. 42, $\mathrm{N}^{\circ} 2$, Wiley.

Mosley, P., J. Hudson y S. Horrel (1987), "Aid, the public sector and the market in less developed economies", The Economic Journal, vol. 97.

Neira, I., E. Vázquez y M. Portela (2009), "An empirical analysis of social capital and economic growth in Europe (1980-2000)", Social Indicators Research, vol. 92, $\mathrm{N}^{\circ} 1$, Springer.

oCDE (Organización para la Cooperación y el Desarrollo Económicos) (2013), "DAC database" [en línea] http://www.oecd.org/dac/ stats/international-development-statistics.htm.

(2001), The Well-being of Nations. The Role of Human and Social Capital, París.

Oorschot, W. y W. Arts (2005), "The social capital of European welfare states: the crowding out hypothesis revisited", Journal of European Social Policy, vol. 15, $\mathrm{N}^{\circ}$ 1, SAGE.

Ostrom, E. (2000), "Social capital. A fad or fundamental concept?", Social Capital: A Multifaceted Perspective, P. Dasgupta e I. Seragilden (ed.), Washington, D.C., Banco Mundial.
Polity IV (2013) [en línea] http://www.systemicpeace.org/inscr/ inscr.htm.

Putnam, R.D. (2000), Bowling Alone: The Collapse and Revival of American Community, Nueva York, Simon \& Schuster. (1995), "Bowling alone: America's declining social capital", Journal of Democracy, vol. 6, $\mathrm{N}^{\circ} 1$.

Putnam, R., R. Leonardi y R. Nanetti (1993), Making Democracy Work: Civic Traditions in Modern Italy, Princeton, Princenton University Press.

Rajan, R. y A. Subramanian (2008), "Aid and growth: what does the cross-country evidence really show?", The Review of Economics and Statistics, vol. 90, $\mathrm{N}^{\circ}$ 4, Cambridge, Massachusetts, The MIT Press.

Rivera-Batiz, F. (2002), "Democracy, governance and economic growth: theory and evidence", Review of Development Economics, vol. 6, $\mathrm{N}^{\circ}$ 2, Wiley.

Sachs, J. (2011), The End of Poverty: How Can we Make It Happen in our Lifetime, Londres, Penguin.

Sargan, J.D. (1958), "The estimaction of economic relationship using instrumental variables", Econometrica, vol. 26, № 3, Nueva York, The Econometric Society.

sCIG (Social Capital Interest Group) (1999), "Social Capital: A Position Paper" [en línea] http://www.ssc.msu.edu/*internat/ soccap/position.htm.

Simon F. y M. McGillivray (2003), "Aid and public sector borrowing in developing countries", Journal of International Development, vol. $15, \mathrm{~N}^{\circ} 8$, Wiley.

Tarp, F. (2006), "Aid and development", Swedish Economic Policy Review, vol. 13, $\mathrm{N}^{\circ}$ 2, Government Offices of Sweden.

Temple, J. (2001), "Growth effects of education and social capital in the oECD countries", OECD Economic Studies, №33, París.

Whiteley, P. (2000), "Economic growth and social capital", Political Studies, vol. 48, $\mathrm{N}^{\circ} 3$, Wiley. 